\title{
FAST APPROXIMATE NEAREST NEIGHBORS WITH AUTOMATIC ALGORITHM CONFIGURATION
}

\author{
Marius Muja, David G. Lowe \\ Computer Science Department, University of British Columbia, Vancouver, B.C., Canada \\ mariusm@cs.ubc.ca,lowe@cs.ubc.ca
}

Keywords: nearest-neighbors search, randomized kd-trees, hierarchical k-means tree, clustering.

\begin{abstract}
For many computer vision problems, the most time consuming component consists of nearest neighbor matching in high-dimensional spaces. There are no known exact algorithms for solving these high-dimensional problems that are faster than linear search. Approximate algorithms are known to provide large speedups with only minor loss in accuracy, but many such algorithms have been published with only minimal guidance on selecting an algorithm and its parameters for any given problem. In this paper, we describe a system that answers the question, "What is the fastest approximate nearest-neighbor algorithm for my data?" Our system will take any given dataset and desired degree of precision and use these to automatically determine the best algorithm and parameter values. We also describe a new algorithm that applies priority search on hierarchical k-means trees, which we have found to provide the best known performance on many datasets. After testing a range of alternatives, we have found that multiple randomized k-d trees provide the best performance for other datasets. We are releasing public domain code that implements these approaches. This library provides about one order of magnitude improvement in query time over the best previously available software and provides fully automated parameter selection.
\end{abstract}

\section{INTRODUCTION}

The most computationally expensive part of many computer vision algorithms consists of searching for the closest matches to high-dimensional vectors. Examples of such problems include finding the best matches for local image features in large datasets (Lowe, 2004; Philbin et al., 2007), clustering local features into visual words using the k-means or similar algorithms (Sivic and Zisserman, 2003), or performing normalized cross-correlation to compare image patches in large datasets (Torralba et al., 2008). The nearest neighbor search problem is also of major importance in many other applications, including machine learning, document retrieval, data compression, bioinformatics, and data analysis.

We can define the nearest neighbor search problem as follows: given a set of points $P=\left\{p_{1}, \ldots, p_{n}\right\}$ in a vector space $X$, these points must be preprocessed in such a way that given a new query point $q \in X$, finding the points in $P$ that are nearest to $q$ can be per- formed efficiently. In this paper, we will assume that $X$ is an Euclidean vector space, which is appropriate for most problems in computer vision. We will describe potential extensions of our approach to general metric spaces, although this would come at some cost in efficiency.

For high-dimensional spaces, there are often no known algorithms for nearest neighbor search that are more efficient than simple linear search. As linear search is too costly for many applications, this has generated an interest in algorithms that perform approximate nearest neighbor search, in which nonoptimal neighbors are sometimes returned. Such approximate algorithms can be orders of magnitude faster than exact search, while still providing nearoptimal accuracy.

There have been hundreds of papers published on algorithms for approximate nearest neighbor search, but there has been little systematic comparison to guide the choice among algorithms and set their internal parameters. One reason for this is that the relative 
performance of the algorithms varies widely based on properties of the datasets, such as dimensionality, correlations, clustering characteristics, and size. In this paper, we attempt to bring some order to these results by comparing the most promising methods on a range of realistic datasets with a wide range of dimensionality. In addition, we have developed an approach for automatic algorithm selection and configuration, which allows the best algorithm and parameter settings to be determined automatically for any given dataset. This approach allows for easy extension if other algorithms are identified in the future that provide superior performance for particular datasets. Our code is being made available in the public domain to make it easy for others to perform comparisons and contribute to improvements.

We also introduce an algorithm which modifies the previous method of using hierarchical k-means trees. While previous methods for searching k-means trees have used a branch-and-bound approach that searches in depth-first order, our method uses a priority queue to expand the search in order according to the distance of each k-means domain from the query. In addition, we are able to reduce the tree construction time by about an order of magnitude by limiting the number of iterations for which the k-means clustering is performed. For many datasets, we find that this algorithm has the highest known performance.

For other datasets, we have found that an algorithm that uses multiple randomized kd-trees provides the best results. This algorithm has only been proposed recently (Silpa-Anan and Hartley, 2004; SilpaAnan and Hartley, 2008) and has not been widely tested. Our results show that once optimal parameter values have been determined this algorithm often gives an order of magnitude improvement compared to the best previous method that used a single kd-tree.

From the perspective of a person using our software, no familiarity with the algorithms is necessary and only some simple library routines are called. The user provides a dataset, and our algorithm uses a cross-validation approach to identify the best algorithm and to search for the optimal parameter values to minimize the predicted search cost of future queries. The user may also specify a desire to accept a less than optimal query time in exchange for reduced memory usage, a reduction in data structure build-time, or reduced time spent on parameter selection.

We demonstrate our approach by matching image patches to a database of 100,000 other patches taken under different illumination and imaging conditions. In our experiments, we show it is possible to obtain a speed-up by a factor of 1,000 times relative to lin- ear search while still identifying $95 \%$ of the correct nearest neighbors.

\section{PREVIOUS RESEARCH}

The most widely used algorithm for nearest-neighbor search is the kd-tree (Freidman et al., 1977), which works well for exact nearest neighbor search in lowdimensional data, but quickly loses its effectiveness as dimensionality increases. Arya et al. (Arya et al., 1998) modify the original kd-tree algorithm to use it for approximate matching. They impose a bound on the accuracy of a solution using the notion of $\varepsilon$ approximate nearest neighbor: a point $p \in X$ is an $\varepsilon$ approximate nearest neighbor of a query point $q \in X$, if $\operatorname{dist}(p, q) \leq(1+\varepsilon) \operatorname{dist}\left(p^{*}, q\right)$ where $p^{*}$ is the true nearest neighbor. The authors also propose the use of a priority queue to speed up the search in a tree by visiting tree nodes in order of their distance from the query point. Beis and Lowe (Beis and Lowe, 1997) describe a similar kd-tree based algorithm, but use a stopping criterion based on examining a fixed number $E_{\text {max }}$ of leaf nodes, which can give better performance than the $\varepsilon$-approximate cutoff.

Silpa-Anan and Hartley (Silpa-Anan and Hartley, 2008) propose the use of multiple randomized $\mathrm{kd}$ trees as a means to speed up approximate nearestneighbor search. They perform only limited tests, but we have found this to work well over a wide range of problems.

Fukunaga and Narendra (Fukunaga and Narendra, 1975 ) propose that nearest-neighbor matching be performed with a tree structure constructed by clustering the data points with the k-means algorithm into $k$ disjoint groups and then recursively doing the same for each of the groups. The tree they propose requires a vector space because they compute the mean of each cluster. Brin (Brin, 1995) proposes a similar tree, called GNAT, Geometric Near-neighbor Access Tree, in which he uses some of the data points as the cluster centers instead of computing the cluster mean points. This change allows the tree to be defined in a general metric space.

Liu et al. (Liu et al., 2004) propose a new kind of metric tree that allows an overlap between the children of each node, called the spill-tree. However, our experiments so far have found that randomized kdtrees provide higher performance while requiring less memory.

Nister and Stewenius (Nister and Stewenius, 2006) present a fast method for nearest-neighbor feature search in very large databases. Their method is based on accessing a single leaf node of a hierarchi- 

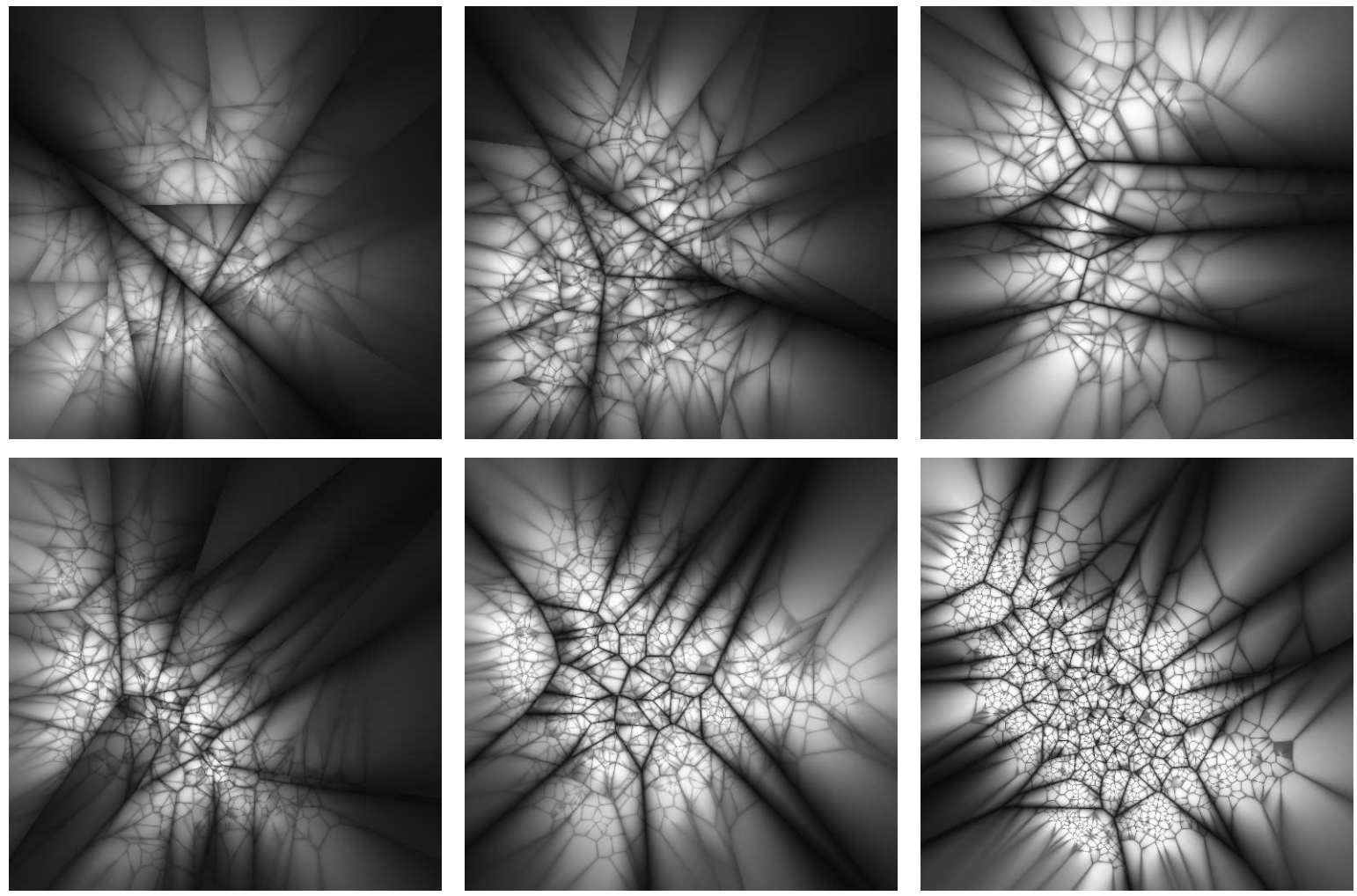

Figure 1: Projections of hierarchical k-means trees constructed using the same 100K SIFT features dataset with different branching factors: $2,4,8,16,32,128$. The projections are constructed using the same technique as in (Schindler et al., 2007). The gray values indicate the ratio between the distances to the nearest and the second-nearest cluster center at each tree level, so that the darkest values (ratio $\approx 1)$ fall near the boundaries between k-means regions.

cal k-means tree similar to that proposed by Fukunaga and Narendra (Fukunaga and Narendra, 1975).

In (Leibe et al., 2006) the authors propose an efficient method for clustering and matching features in large datasets. They compare several clustering methods: k-means clustering, agglomerative clustering, and a combined partitional-agglomerative algorithm. Similarly, (Mikolajczyk and Matas, 2007) evaluates the nearest neighbor matching performance for several tree structures, including the kd-tree, the hierarchical k-means tree, and the agglomerative tree. We have used these experiments to guide our choice of algorithms.

\section{FINDING FAST APPROXIMATE NEAREST NEIGHBORS}

We have compared many different algorithms for approximate nearest neighbor search on datasets with a wide range of dimensionality. The accuracy of the approximation is measured in terms of precision, which is defined as the percentage of query points for which the correct nearest neighbor is found. In our experiments, one of two algorithms obtained the best performance, depending on the dataset and desired precision. These algorithms used either the hierarchical $\mathrm{k}$-means tree or multiple randomized kd-trees. In this section, we will begin by describing these algorithms. We give comparisons to some other approaches in the experiments section.

\subsection{The randomized kd-tree algorithm}

The classical kd-tree algorithm (Freidman et al., 1977) is efficient in low dimensions, but in high dimensions the performance rapidly degrades. To obtain a speedup over linear search it becomes necessary to settle for an approximate nearest-neighbor. This improves the search speed at the cost of the algorithm not always returning the exact nearest neighbors.

Silpa-Anan and Hartley (Silpa-Anan and Hartley, 2008) have recently proposed an improved version of the kd-tree algorithm in which multiple randomized kd-trees are created. The original kd-tree algorithm 
splits the data in half at each level of the tree on the dimension for which the data exhibits the greatest variance. By comparison, the randomized trees are built by choosing the split dimension randomly from the first $D$ dimensions on which data has the greatest variance. We use the fixed value $D=5$ in our implementation, as this performs well across all our datasets and does not benefit significantly from further tuning.

When searching the trees, a single priority queue is maintained across all the randomized trees so that search can be ordered by increasing distance to each bin boundary. The degree of approximation is determined by examining a fixed number of leaf nodes, at which point the search is terminated and the best candidates returned. In our implementation the user specifies only the desired search precision, which is used during training to select the number of leaf nodes that will be examined in order to achieve this precision.

\subsection{The hierarchical k-means tree algorithm}

The hierarchical k-means tree is constructed by splitting the data points at each level into $K$ distinct regions using a k-means clustering, and then applying the same method recursively to the points in each region. We stop the recursion when the number of points in a region is smaller than $K$. Figure 1 shows projections of several hierarchical k-means trees constructed from 100K SIFT features using different branching factors.

We have developed an algorithm that explores the hierarchical k-means tree in a best-bin-first manner, by analogy to what has been found to improve the exploration of the kd-tree. The algorithm initially performs a single traversal through the tree and adds to a priority queue all unexplored branches in each node along the path. Next, it extracts from the priority queue the branch that has the closest center to the query point and it restarts the tree traversal from that branch. In each traversal the algorithm keeps adding to the priority queue the unexplored branches along the path. The degree of approximation is specified in the same way as for the randomized kd-trees, by stopping the search early after a predetermined number of leaf nodes (dataset points) have been examined. This parameter is set during training to achieve the userspecified search precision.

We have experimented with a number of variations, such as using the distance to the closest Voronoi border instead of the distance to the closest cluster center, using multiple randomized k-means trees, or using agglomerative clustering as proposed by Leibe et al. (Leibe et al., 2006). However, these were found not to give improved performance.

\subsection{Automatic selection of the optimal algorithm}

Our experiments have revealed that the optimal algorithm for fast approximate nearest neighbor search is highly dependent on several factors such as the structure of the dataset (whether there is any correlation between the features in the dataset) and the desired search precision. Additionally, each algorithm has a set of parameters that have significant influence on the search performance. These parameters include the number of randomized trees to use in the case of $\mathrm{kd}$ trees or the branching factor and the number of iterations in the case of the hierarchical k-means tree.

By considering the algorithm itself as a parameter of a generic nearest neighbor search routine, the problem is reduced to determining the parameters that give the best solution. This is an optimization problem in the parameter space. The cost is computed as a combination of the search time, tree build time, and tree memory overhead. Depending on the application, each of these three factors has a different importance: in some cases we don't care much about the tree build time (if we will build the tree only once and use it for a large number of queries), while in other cases both the tree build time and search time must be small (if the tree is built on-line and searched a small number of times). There are also situations when we wish to limit the memory overhead. We control the relative importance of each of these factors by using a buildtime weight, $w_{b}$, and a memory weight, $w_{m}$, to compute the overall cost:

$$
\text { cost }=\frac{s+w_{b} b}{\left(s+w_{b} b\right)_{\mathrm{opt}}}+w_{m} m
$$

where $s$ represents the search time for the number of vectors in the sample dataset, $b$ represents the tree build time, and $m=m_{t} / m_{d}$ represents the ratio of memory used for the tree $\left(m_{t}\right)$ to the memory used to store the data $\left(m_{d}\right)$.

The build-time weight $\left(w_{b}\right)$ controls the importance of the tree build time relative to the search time. Setting $w_{b}=0$ means that we want the fastest search time and don't care about the tree build time, while $w_{b}=1$ means that the tree build time and the search time have the same importance. Similarly, the memory weight $\left(w_{m}\right)$ controls the importance of the memory overhead compared to the time overhead. The time overhead is computed relative to the optimum time cost $\left(s+w_{b} b\right)_{\text {opt }}$, which is defined as the optimal search and build time if memory usage is not a factor. Therefore, setting $w_{m}=0$ will choose the algorithm and parameters that result in the fastest search 
and build time without any regard to memory overhead, while setting $w_{m}=1$ will give equal weight to a given percentage increase in memory use as to the same percentage increase in search and build time.

We choose the best nearest neighbor algorithm and the optimum parameters in a two step approach: a global exploration of the parameter space followed by a local tuning of the best parameters. Initially, we sample the parameter space at multiple points and choose those values that minimize the cost of equation 1 . For this step we consider using $\{1,4,8,16,32\}$ as the number of random kd-trees, $\{16,32,64,128,256\}$ as the branching factor for the k-means tree and $\{1,5,10,15\}$ as the number of $\mathrm{k}$-means iterations. In the second step we use the Nelder-Mead downhill simplex method to further locally explore the parameter space and fine-tune the best parameters obtained in the first step. Although this does not guarantee a global minimum, our experiments have shown that the parameter values obtained are close to optimum.

The optimization can be run on the full dataset or just on a fraction of the dataset. Using the full dataset gives the most accurate results but in the case of large datasets it may take more time than desired. An option is provided to use just a fraction of the dataset to select parameter values. We have found that using just one tenth of the dataset typically selects parameter values that still perform close to the optimum on the full dataset. The parameter selection needs only be performed once for each type of dataset, and our library allows these values to be saved and applied to all future datasets of the same type.

\section{EXPERIMENTS}

\subsection{Randomized kd-trees}

Figure 2 shows the value of searching in many randomized kd-trees at the same time. It can be seen that performance improves with the number of randomized trees up to a certain point (about 20 random trees in this case) and that increasing the number of random trees further leads to static or decreasing performance. The memory overhead of using multiple random trees increases linearly with the number of trees, so the cost function may choose a lower number if memory usage is assigned importance.

\subsection{Hierarchical k-means tree}

The hierarchical k-means tree algorithm has the highest performance for some datasets. However, one disadvantage of this algorithm is that it often has a

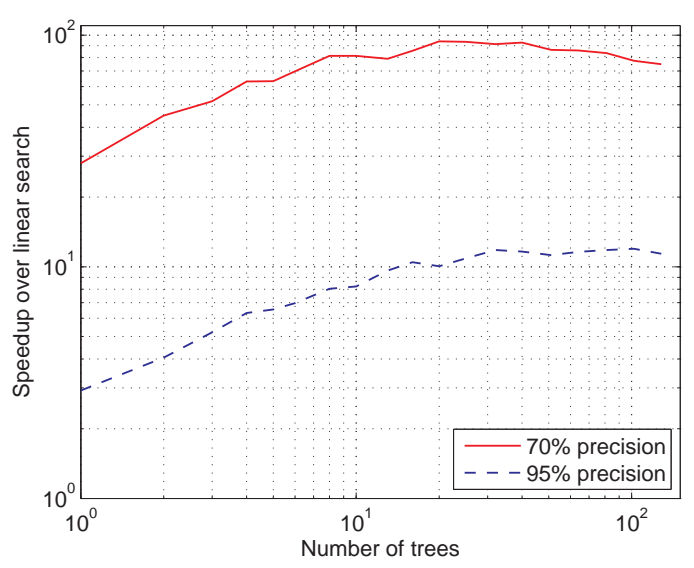

Figure 2: Speedup obtained by using multiple random kdtrees (100K SIFT features dataset)

higher tree-build time than the randomized kd-trees. The build time can be reduced significantly by doing a small number of iterations in the k-means clustering stage instead of running it until convergence. Figure 3 shows the performance of the tree constructed using a limited number of iterations in the k-means clustering step relative to the performance of the tree when the $\mathrm{k}$-means clustering is run until convergence. It can be seen that using as few as 7 iterations we get more than $90 \%$ of the nearest-neighbor performance of the tree constructed using full convergence, but requiring less than $10 \%$ of the build time.

When using zero iterations in the k-means clustering we obtain the more general GNAT tree of (Brin, 1995), which assumes that the data lives in a generic metric space, not in a vector space. However figure 3(a) shows that the search performance of this tree is worse than that of the hierarchical k-means tree (by factor of 5).

\subsection{Data dimensionality.}

Data dimensionality is one of the factors that has a great impact on the nearest neighbor matching performance. Figure 4(a) shows how the search performance degrades as the dimensionality increases. The datasets each contain $10^{5}$ vectors whose values are randomly sampled from the same uniform distribution. These random datasets are one of the most difficult problems for nearest neighbor search, as no value gives any predictive information about any other value. As can be seen in figure 4(a), the nearestneighbor searches have a low efficiency for higher dimensional data (for $68 \%$ precision the approximate search speed is no better than linear search when the number of dimensions is greater than 800). However 


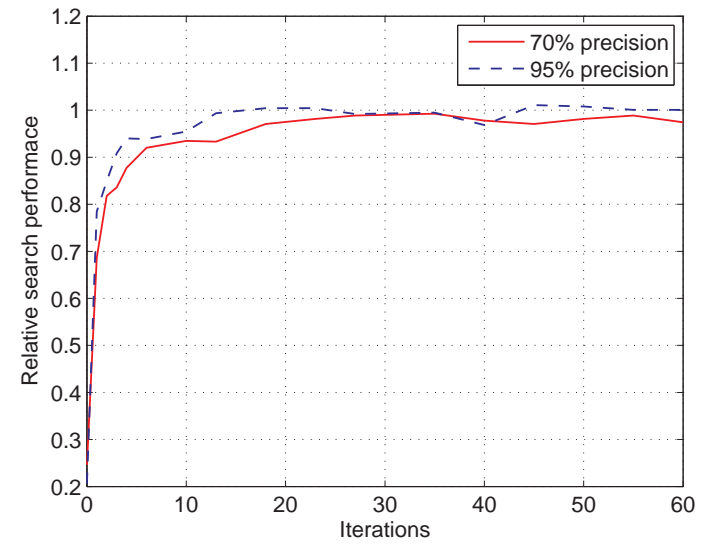

(a)

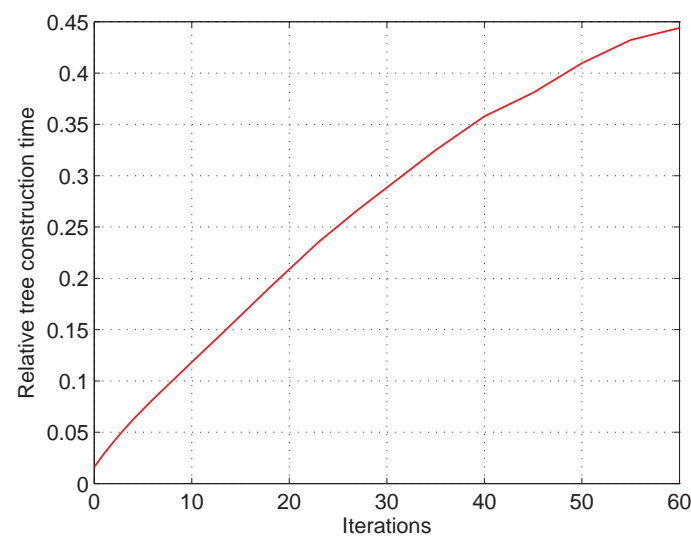

(b)

Figure 3: The influence that the number of k-means iterations has on the search time efficiency of the k-means tree (a) and on the tree construction time (b) (100K SIFT features dataset)

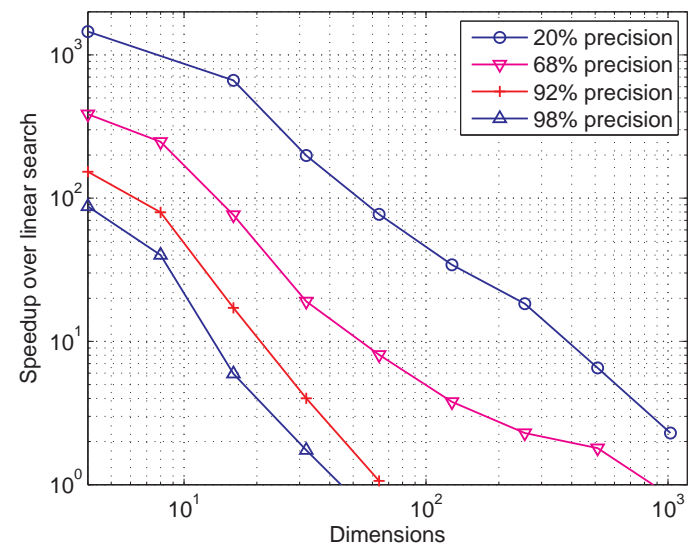

(a) Random vectors

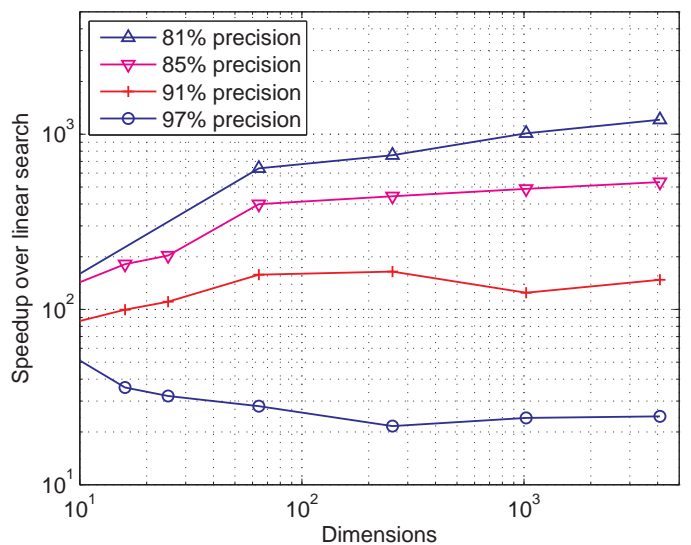

(b) Image patches

Figure 4: Search efficiency for data of varying dimensionality. The random vectors (a) represent the hardest case in which dimensions have no correlations, while most real-world problems behave more like the image patches (b)

real world datasets are normally much easier due to correlations between dimensions.

The performance is markedly different for many real-world datasets. Figure 4(b) shows the speedup as a function of dimensionality for the Winder/Brown image patches ${ }^{1}$ resampled to achieve varying dimensionality. In this case however, the speedup does not decrease with dimensionality, it's actually increasing for some precisions. This can be explained by the fact that there exists a strong correlation between the dimensions, so that even for 64x64 patches (4096 dimensions), the similarity between only a few dimensions provides strong evidence for overall patch similarity.

\footnotetext{
${ }^{1} \mathrm{http}: / /$ phototour.cs.washington.edu/patches/default.htm
}

Figure 5 shows four examples of queries on the Trevi dataset of patches for different patch sizes.

\subsection{Search precision.}

The desired search precision determines the degree of speedup that can be obtained with any approximate algorithm. Looking at figure 6(b) (the sift1M dataset) we see that if we are willing to accept a precision as low as $60 \%$, meaning that $40 \%$ of the neighbors returned are not the exact nearest neighbors, but just approximations, we can achieve a speedup of three orders of magnitude over linear search (using the multiple randomized kd-trees). However, if we require a precision greater then $90 \%$ the speedup is smaller, 

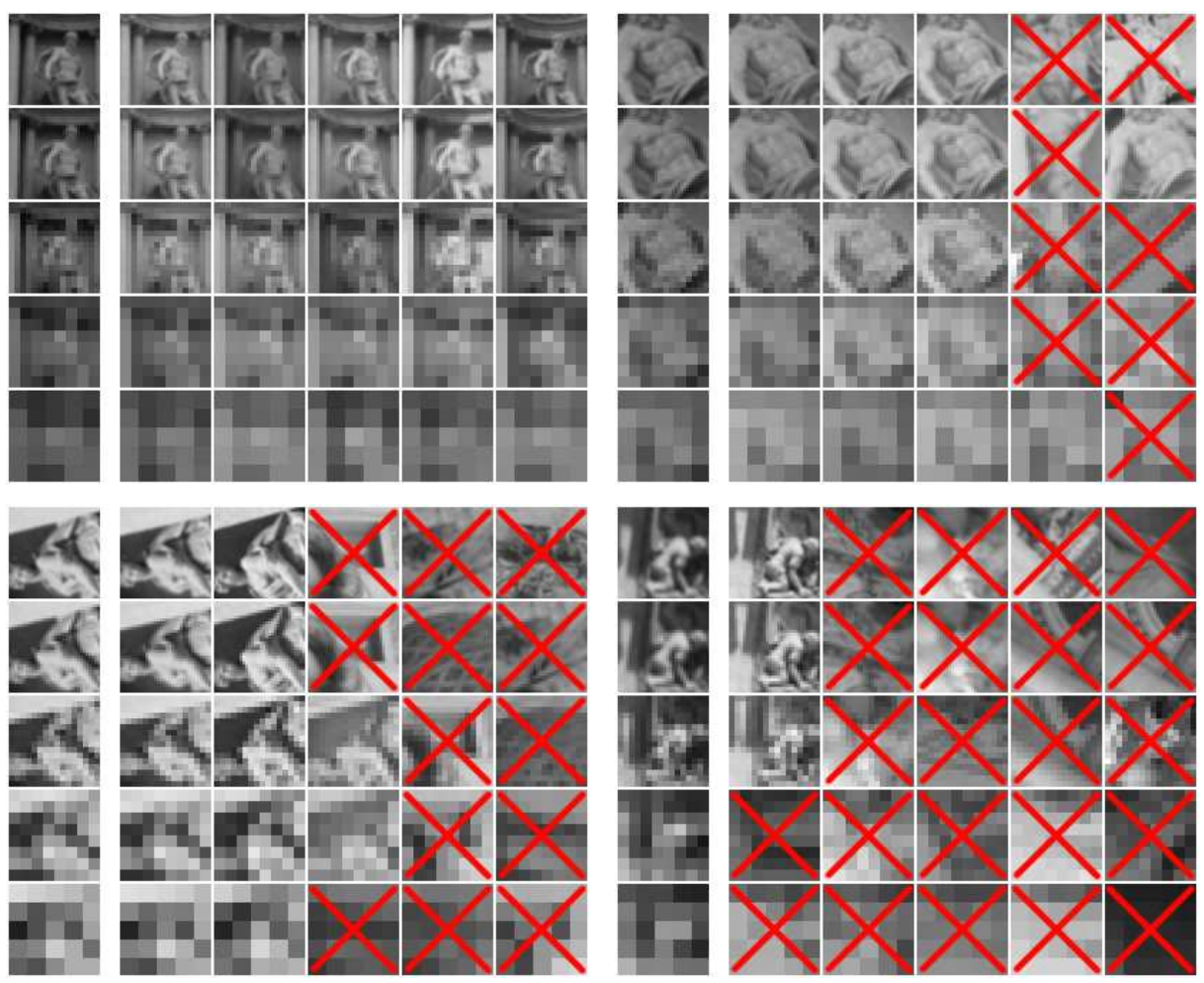

Figure 5: Examples of querying the Trevi Fountain patch dataset using different patch sizes. The query patch is on the left of each panel, while the following 5 patches are the nearest neighbors from a set of 100,000 patches. Incorrect matches are shown with an $\mathrm{X}$.

less than 2 orders of magnitude (using the hierarchical k-means tree).

We use several datasets of different dimensions for the experiments in figure 6 . We construct a $100 \mathrm{~K}$ and 1 million SIFT features dataset by randomly sampling a dataset of over 5 million SIFT features extracted from a collection of CD cover images (Nister and Stewenius, 2006) ${ }^{2}$. These two datasets obtained by random sampling have a relatively high degree of difficulty in terms of nearest neighbour matching, because the features they contain usually do not have "true" matches between them. We also use the entire 31 million feature dataset from the same source for the experiment in figure 6(b). Additionally we use the patches datasets described in subsection 4.3 and another $100 \mathrm{~K}$ SIFT features dataset obtained from a set of images forming a panorama.

We compare the two algorithms we found to be the best at finding fast approximate nearest neighbors (the multiple randomized kd-trees and the hierarchical kmeans tree) with existing approaches, the ANN (Arya et al., 1998) and LSH algorithms (Andoni, 2006) ${ }^{3}$ on

\footnotetext{
${ }^{2}$ http://www.vis.uky.edu/ stewe/ukbench/data/

${ }^{3}$ We have used the publicly available implementations
}

the first dataset of 100,000 SIFT features. Since the LSH implementation (the $\mathrm{E}^{2} \mathrm{LSH}$ package) solves the $R$-near neighbor problem (finds the neighbors within a radius $R$ of the query point, not the nearest neighbors), to find the nearest neighbors we have used the approach suggested in the $E^{2}$ LSH's user manual: we compute the $R$-near neighbors for increasing values of $R$. The parameters for the LSH algorithm were chosen using the parameter estimation tool included in the $E^{2} \mathrm{LSH}$ package. For each case we have computed the precision achieved as the percentage of the query points for which the nearest neighbors were correctly found. Figure 6(a) shows that the hierarchical k-means algorithm outperforms both the ANN and LSH algorithms by about an order of magnitude. The results for ANN are consistent with the experiment in figure 2, as ANN uses only a single kd-tree and does not benefit from the speedup due to using multiple randomized trees.

Figure 6(b) shows the performance of the randomized kd-trees and the hierarchical k-means on datasets of different sizes. The figure shows that the two algo-

of ANN (http://www.cs.umd.edu/ mount/ANN/) and LSH (http://www.mit.edu/ andoni/LSH/) 


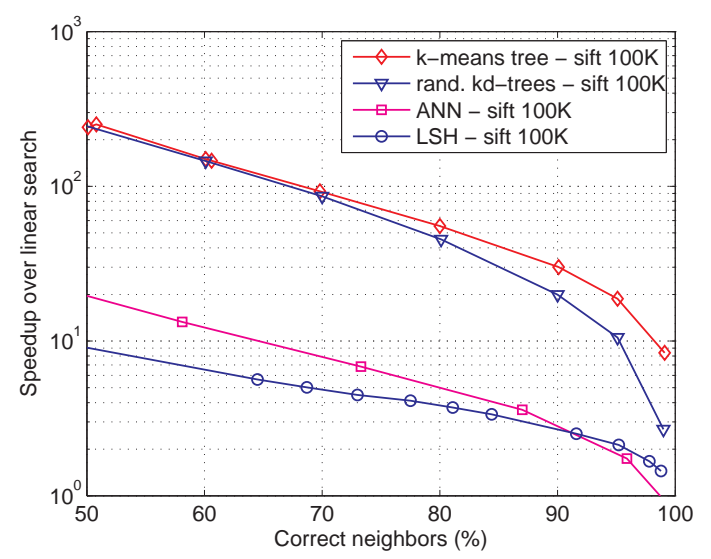

(a)

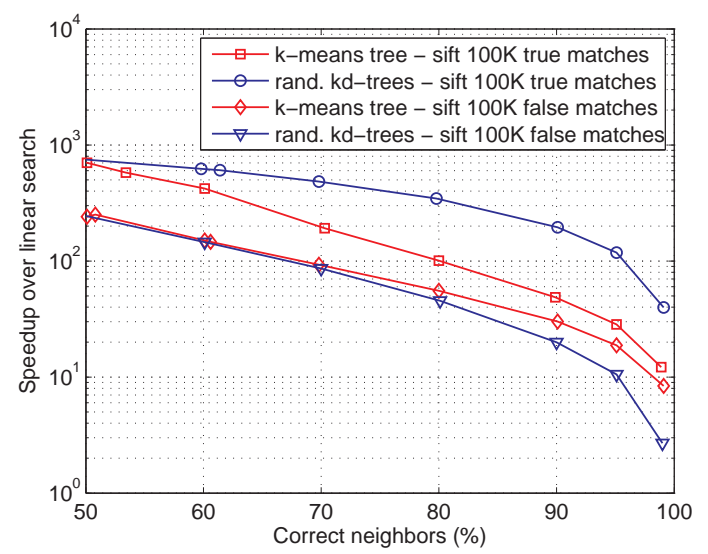

(c)

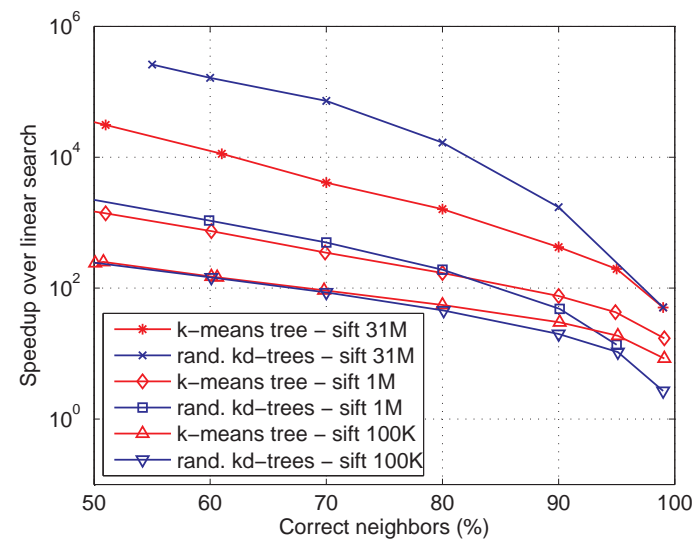

(b)

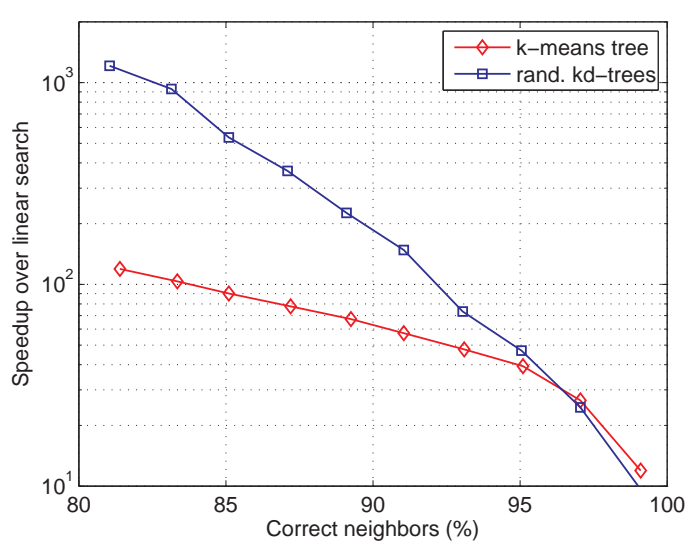

(d)

Figure 6: Search efficiency. (a) Comparison of different algorithms. (b) Search speedup for different dataset sizes. (c) Search speedup when the query points don't have "true" matches in the dataset vs the case when they have. (d) Search speedup for the Trevi Fountain patches dataset

rithms scale well with the increase in the dataset size, having the speedup over linear search increase with the dataset size.

Figure 6(c) compares the performance of nearest neighbor matching when the dataset contains true matches for each feature in the test set to the case when it contains false matches. In this experiment we used the two 100K SIFT features datasets described above. The first is randomly sampled from a 5 million SIFT features dataset and it contains false matches for each feature in the test set. The second contains SIFT features extracted from a set of images forming a panorama. These features were extracted from the overlapping regions of the images, and we use only those that have a true match in the dataset. Our experiments showed that the randomized kd-trees have a significantly better performance for true matches, when the query features are likely to be significantly closer than other neighbors. Similar results were reported in (Mikolajczyk and Matas, 2007).

Figure 6(d) shows the difference in performance between the randomized kd-trees and the hierarchical $\mathrm{k}$-means tree for one of the Winder/Brown patches dataset. In this case, the randomized kd-trees algorithm clearly outperforms the hierarchical k-means algorithm everywhere except for precisions very close to $100 \%$. It appears that the kd-tree works much better in cases when the intrinsic dimensionality of the data is much lower than the actual dimensionality, presumably because it can better exploit the correlations among dimensions. However, Figure 6(b) shows that the k-means tree can perform better for other datasets (especially for high precisions). This shows the importance of performing algorithm selection on each dataset. 
Table 1: The algorithms chosen by our automatic algorithm and parameter selection procedure (sift100K dataset). The "Algorithm" column shows the algorithm chosen and its optimum parameters (number of random trees in case of the kd-tree; branching factor and number of iterations for the k-means tree), the "Dist. Error" column shows the mean distance error compared to the exact nearest neighbors, the "Search Speedup" shows the search speedup compared to linear search, the "Memory Used" shows the memory used by the tree(s) as a fraction of the memory used by the dataset and the "Build Time" column shows the tree build time as a fraction of the linear search time for the test set.

\begin{tabular}{|c|c|c|c|c|c|c|c|}
\hline $\operatorname{Pr} .(\%)$ & $w_{b}$ & $w_{m}$ & Algorithm & $\begin{array}{l}\text { Dist. } \\
\text { Error }\end{array}$ & $\begin{array}{c}\text { Search } \\
\text { Speedup }\end{array}$ & $\begin{array}{l}\text { Memory } \\
\text { Used }\end{array}$ & $\begin{array}{l}\text { Build } \\
\text { Time }\end{array}$ \\
\hline \multirow{6}{*}{$60 \%$} & 0 & 0 & k-means, 16,15 & 0.096 & 181.10 & 0.51 & 0.58 \\
\hline & 0 & 1 & k-means, 32, 10 & 0.058 & 180.9 & 0.37 & 0.56 \\
\hline & 0.01 & 0 & k-means, 16,5 & 0.077 & 163.25 & 0.50 & 0.26 \\
\hline & 0.01 & 1 & kd-tree, 4 & 0.041 & 109.50 & 0.26 & 0.12 \\
\hline & 1 & 0 & kd-tree, 1 & 0.044 & 56.87 & 0.07 & 0.03 \\
\hline & $*$ & $\infty$ & kd-tree, 1 & 0.044 & 56.87 & 0.07 & 0.03 \\
\hline \multirow{6}{*}{$90 \%$} & 0 & 0 & k-means, 128,10 & 0.008 & 31.67 & 0.18 & 1.82 \\
\hline & 0 & 1 & k-means, 128,15 & 0.007 & 30.53 & 0.18 & 2.32 \\
\hline & 0.01 & 0 & k-means, 32, 5 & 0.011 & 29.47 & 0.36 & 0.35 \\
\hline & 1 & 0 & k-means, 16,1 & 0.016 & 21.59 & 0.48 & 0.10 \\
\hline & 1 & 1 & kd-tree, 1 & 0.005 & 5.05 & 0.07 & 0.03 \\
\hline & $*$ & $\infty$ & kd-tree, 1 & 0.005 & 5.05 & 0.07 & 0.03 \\
\hline
\end{tabular}

\subsection{Automatic algorithm and parameter selection.}

In table 1 , we show the results from running the parameter selection procedure described in 3.3 on the dataset containing $100 \mathrm{~K}$ random sampled SIFT features. We have used two different search precisions $(60 \%$ and $90 \%)$ and several combinations of the tradeoff factors $w_{b}$ and $w_{m}$. For the build time weight, $w_{b}$, we used three different possible values: 0 representing the case where we don't care about the tree build time, 1 for the case where the tree build time and search time have the same importance and 0.01 representing the case where we care mainly about the search time but we also want to avoid a large build time. Similarly, the memory weight was chosen to be 0 for the case where the memory usage is not a concern, $\infty$ representing the case where the memory use is the dominant concern and 1 as a middle ground between the two cases.

Examining table 1 we can see that for the cases when the build time or the memory overhead had the highest weight, the algorithm chosen was the kd-tree with a single tree because it is both the most memory efficient and the fastest to build. When no importance was given to the tree build time and the memory overhead the algorithm chosen was k-means, as confirmed by the plots in figure $6(\mathrm{~b})$. The branching factors and the number of iterations chosen for the k-means algorithm depend on the search precision and the tree build time weight: higher branching factors proved to have better performance for higher precisions and the tree build time increases when the branching factor or the number of iterations increase.

\section{CONCLUSIONS}

In the approach described in this paper, automatic algorithm configuration allows a user to achieve high performance in approximate nearest neighbor matching by calling a single library routine. The user need only provide an example of the type of dataset that will be used and the desired precision, and may optionally specify the importance of minimizing memory or build time rather than just search time. All remaining steps of algorithm selection and parameter optimization are performed automatically.

In our experiments, we have found that either of two algorithms can have the best performance, depending on the dataset and desired precision. One of these is an algorithm we have developed that combines two previous approaches: searching hierarchical k-means trees with a priority search order. The second method is to use multiple randomized $\mathrm{kd}$ trees. We have demonstrated that these can speed the matching of high-dimensional vectors by up to several orders of magnitude compared to linear search.

The use of automated algorithm configuration will make it easy to incorporate any new algorithms that are found in the future to have superior performance for particular datasets. We intend to keep adding new datasets to our website and provide test results for further algorithms. Our public domain library will 
enable others to perform detailed comparisons with new approaches and contribute to the extension of this software.

\section{ACKNOWLEDGMENTS}

This research has been supported by the Natural Sciences and Engineering Research Council of Canada and by the Canadian Institute for Advanced Research. We wish to thank Matthew Brown, Richard Hartley, Scott Helmer, Hoyt Koepke, Sancho McCann, Panu Turcot and Andrew Zisserman for valuable discussions and usefull feedback regarding this work.

\section{REFERENCES}

Andoni, A. (2006). Near-optimal hashing algorithms for approximate nearest neighbor in high dimensions. Proceedings of the 47th Annual IEEE Symposium on Foundations of Computer Science (FOCS'06), pages 459-468.

Arya, S., Mount, D. M., Netanyahu, N. S., Silverman, R., and Wu, A. Y. (1998). An optimal algorithm for approximate nearest neighbor searching in fixed dimensions. Journal of the ACM, 45:891-923.

Beis, J. S. and Lowe, D. G. (1997). Shape indexing using approximate nearest-neighbor search in high dimensional spaces. In $C V P R$, pages 1000-1006.

Brin, S. (1995). Near neighbor search in large metric spaces. In $V L D B$, pages 574-584.

Freidman, J. H., Bentley, J. L., and Finkel, R. A. (1977). An algorithm for finding best matches in logarithmic expected time. ACM Trans. Math. Softw., 3:209-226.
Fukunaga, K. and Narendra, P. M. (1975). A branch and bound algorithm for computing $\mathrm{k}$-nearest neighbors. IEEE Trans. Comput., 24:750-753.

Leibe, B., Mikolajczyk, K., and Schiele, B. (2006). Efficient clustering and matching for object class recognition. In $B M V C$.

Liu, T., Moore, A., Gray, A., and Yang, K. (2004). An investigation of practical approximate nearest neighbor algorithms. In Neural Information Processing Systems.

Lowe, D. G. (2004). Distinctive image features from scaleinvariant keypoints. Int. Journal of Computer Vision, 60:91-110.

Mikolajczyk, K. and Matas, J. (2007). Improving descriptors for fast tree matching by optimal linear projection. In Computer Vision, 2007. ICCV 2007. IEEE 11th International Conference on, pages 1-8.

Nister, D. and Stewenius, H. (2006). Scalable recognition with a vocabulary tree. In CVPR, pages 2161-2168.

Philbin, J., Chum, O., Isard, M., Sivic, J., and Zisserman, A. (2007). Object retrieval with large vocabularies and fast spatial matching. In CVPR.

Schindler, G., Brown, M., and Szeliski, R. (2007). Cityscale location recognition. In $C V P R$, pages $1-7$.

Silpa-Anan, C. and Hartley, R. (2004). Localization using an imagemap. In Australasian Conference on Robotics and Automation.

Silpa-Anan, C. and Hartley, R. (2008). Optimised KD-trees for fast image descriptor matching. In $C V P R$.

Sivic, J. and Zisserman, A. (2003). Video Google: A text retrieval approach to object matching in videos. In $I C C V$.

Torralba, A., Fergus, R., and Freeman, W. T. (2008). 80 million tiny images: A large data set for nonparametric object and scene recognition. IEEE Transactions on Pattern Analysis and Machine Intelligence, 30(11):1958-1970. 